This report was prepared as an account of work sponsored by the United States Government. Neither the United States nor the United States Department of Energy, nor any of their employees, nor any of their contractors, subcontractors, or their employees, makes any warranty, express or 1mplied, or assumeg any legal liability or respongibility for the accuracy, completeness or usefulness of any information, apparatus, product or process disclosed, or represents that 1 ts use would not infringe privately cined rights.

Printed in the Jnited states of America

Avatlable from:

National Technical Information Service

U.S. Departront of Commerce

5285 Port Royal hoad

Springfield, Virginia 22161

Price Printed Copy $\$ \ldots$ Microfiche $\$ 4.50$

Pagea

$1=25$

25-50

$51-75$

$76-100$

$101-125$

126-150

$151-175$

$176-200$

201-225

226-250

$251-275$

$276-300$

$301-325$

$326-350$

$351-375$

$376-400$

$401-425$

$426-450$

$451-475$

476-500

$500-525$

526-550

551-575

567-600

RIIS

Seling Price

$\$ 7.00$

$\$ 8.50$

$\$ 10.00$

$\$ 11.50$

$\$ 13.00$

$\$ 14.50$

$\$ 16.00$

$\$ 17.50$

$\$ 19.00$

$\$ 20.50$

$\$ 72.00$

$\$ 23.50$

$\$ 25.00$

$\$ 26.50$

$\$ 28.00$

$\$ 29.50$

$\$ 31.00$

$\$ 32.50$

$\$ 34.00$

$\$ 35.50$

$\$ 37.00$

$\$ 38.50$

$\$ 40.00$

$\$ 41.50$
For documinta over 600 pages, add $\$ 1.50$ for each additional 25-page increment. 


\title{
OBSERVATION OF IMPURITY ACCIMULATION AND CONCURRENT \\ IMPURITY INFLUX IN PBX
}

\author{
S.S. Sesnic, R.J. Fonok, K. Ida, ${ }^{t}$ K. Bol, P. Couture, ${ }^{+t}$ \\ G. Gammel, R. Kaita, S, Kaye, H. Kugel, B. LéBlanc, W. Morris, ${ }^{++t}$ \\ M. Okabayashi, S. Paul, E.T. Powell, M. Reusch, and H. Takahashi \\ Plasma Physics Laboratory, princeton university \\ princeton, N.J, 08540
}

\begin{abstract}
Impurity studies in $L-$ and H-mode discharges in PBX have shown that both types of discharges can evolve into either an inpurity arcumulative or nonaccumulative case. In a typical accumulative discharge, zeff peaks in the center to values of about 5. The central metallic densities can be high, $n_{\text {met }} / \mathrm{n}_{\mathrm{e}} \approx 0.01$, resulting in central radiated power densities in excess of 1 w/cm ${ }^{3}$, consigtent with bolometric estimates. The radial profiles of metals ohtained independently from the line radiation in the soft $x-r a y$ and the vuv regions are very peaked. Concurrent with the peaking, an increase in the impurity influx coming from the edge of the plasma is observed. At the beginning of the accumulation phase the inward particle flux for titanium has values of $6 \times 10^{10}$ and $10 \times 10^{10}$ partioles $/ \mathrm{cm}^{2} \mathrm{~s}$ at minor radii of 6 and $17 \mathrm{~cm}$. At the end of the accumulation phase, this particle flux is strongly increased to values of $3 \times 10^{12}$ and $1 \times 10^{12}$ particles/cmas. This increased flux is mainly due to influx from the edge of the plasma and to a lesser extent due to increased convective transport. Using the measured particle fiux, an estimate of the diffusion coefficient $D$ and the convective velocity $\checkmark$ is obtained.
\end{abstract}

$t$ University of Tokyo, Nagoya, Japan

+ Institut de rechezche d'Hydro-Ouebec

ttt Belliol college, oxford university, U.K.

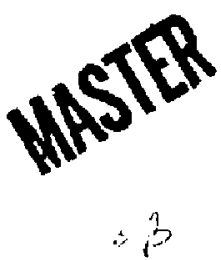




\section{Introduction}

Impurity studies in a tokamak plasma continue to be an integral component of general tokamak investigations for several reasons. For example, radiation losses can impair the confinement and stability of plasmas, and the hydrogen concentration can be diluted by significant impurity densities, resulting in a lower fusion rate. A considerable amount of effort has been expended in studying various facets of tokamak impurity behavior, as described in the excellent review on impurities in tokamaks by R.C. Isler [1]. In this paper we are concerned with one small part of the more general impurity problem: with the flux, accumulation, and transport of metallic impurities in neutral-beam-heated plasmas. The general feature of inpurity accumulation in ohmically heated plasmas, inclioing the facts that accumulation can be prevented by high gas puff rates, only medium-z impurities appear to accumulate strongly, and sawtcoth oscillations can mitigate effects of accumulation by redistribution of the centrally peaked impurities, were all reported in the seminal tork on high density plasmas in the Pulsator tokamak [2]. Most recently, in ASDEx burst-free H-mode discharges a strong accumulation of iron in the center of the plasma was observed [3]. At the same time, impurity accumulation, sastooth-driven redistribution and transport coefficient measurements have received considerable attention in ohmic- and neutrai-beam-heated PBX discharges [4-6], while accumulation and subsequent sawtooth redistribution have been studied in the Alcator-C experiment during silicon injection [7] and peliet fuelling experiments [8]. Even when the steady-state phase of large tokamak discharges does not show strong accumulation, the discharge is usually observed to display accumulation and strong MHD activity early in the discharge, leading to a giant sawtooth crash with "pumpout of the peaked impurities at the onset of regular sawteeth 
$[4,9]$. This wide variety of effects and often conflicting results from different experiments point to the continuing importance of the study of impurity transport in tokamaks.

As reported in detail elsewhere $[5,6]$, PEX discharges of ten display strong accumulation of impurities in the discharge center. In this paper, we discuss evidence for an increased impurity influx leading to the accumulation phase, and show that a novel analysis of radially resolved soft $x$-ray spectra support the earlier conclusions of low transport coefficients in the discharge center [5]. Section II of this paper describes the experimental conditions and the diagnostics utilized in these studies. In the third part, we discuss the medium-z impurity influx and accumulation in neutral-beam-heated f-mode discharges in PBX, while Sec. IV presents an analysis of impurity transport in an acoumulating discharge.

\section{Description of Experiment and Diagnostics}

The Princeton Beta Experiment (PBX) is a neutral-beam-heated tokamak experiment devoted primarily to investigations of high beta plasmas. To facilitate the achievement of high $B$, the discharge cross section has a kidney bean shape, which is produced by an additional curcent-carrying "pusher" coil located on the inside midplane of the vacuum chamber [10]. To improve the avisymmetric stability of the discharge, the plasma is surrounded by nickelplated aluminum plates, which results in nickel being the predominant metallic impurity in the plasma. Depending on the position of the $x$-poinc of the separatrix, the beam-heated discharge had characteristics of either an L-mode ( $\mathrm{X}$-point outside the conducting wall) or an H-mode ( $\mathrm{X}$-point insile the conducting wall) plasma. The minor and major radii varied between 30 to $37 \mathrm{~cm}$ and 143 to $153 \mathrm{~cm}$, respectively. The indentation varied between 0 and 0.2 and 
elongation from 1 to 1.8 . The toroidal magnetic field was relatively 10 w 10.8 to $2.0 \mathrm{~T}$, and the plasma current had values between 250 and $600 \mathrm{kA}$. AIthough the primary objective of PBX was the study of the high $\beta$ regime, an effort was made to understand impurity transport, since impurity fluxes and content very of ten impaired the sustainnent of high $B$ investigations.

PBX was also well suited for impurity transport studies becauge i's had a comprehensive set of diagnostics appropriate for impurity investigation. A multichannel visible bremsstrahlung detector array provided time-resolved $z_{\text {ef } f}$ profiles. SPRED, a multichannel vUV spectrometer, yielded absolute intensities of various impurity lines simultaneously, covering the wavelength rerion between $95 \&$ to 1100 \& wi.th two different diffraction gratings. It was used to measure the impurity influx (lower charge states) and impurity densities (highel charge states) with a time resolution of $20 \mathrm{~ms}$. Two multichannel bolometer arrays provided radiation profiles [11]. Five spatial channels of soft $X$-ray pulse-height analyzers (PHA), two of which were also scannable in minor radius, were used to obtain profiles of the electron temperature $T_{e}(r, t), z_{\text {eff }}(r, t)$, and the metallic impurity densities, $n_{z}(r, t)$, with a variable time resolution which was usually set at 25 ms in these investigarions. Vertical and horizontal multichannel soft $x$-ray diode arrays, normally used for Mirl studies, provided additional measures of the inpurity influx and accumulation, Detailed observation of impurity behavior in PBX from these and other diagnostics are reported elsewhere $[5,6]$ and we concentrate here mainly on information obtained by additional analysis of the data from the soft $x$-ray diagnostics. 
III. Impurity Influx, Accumulation, and Consequent Thermal Collapse The discharges considered here had a plasma current of $300 \mathrm{kA}$, an indentation of 0.1 , central elongation of 41,33 , and a working gas of either $H$ or $D$ heated with $D^{\circ}$ neutral beams with $P_{i n j} \approx 2-5$ MW. Depending on the positioning of the $\mathrm{X}$-point, these discharges accessed either L-mode or $\mathrm{H}-$ mode confinement operating regimes [10] and both types of impurity behavior accumulation or no accumulation - occurred in both I-mode and H-mode discharges [6]. The emphasis in this paper is on the H-mode accumulation case, because the transport can be most readily analyzed in this case. Such discharges were characterized by a thermal collapse of the central plasma, preceded by strong medium-z inpurity influx and accumulation. Some of the plasma parameters for an h-mode discharge with accumulation are shown in Fig. 1. Note that although the density is steadily rising, the central value of the total $z_{\text {eff }}(0)$ and the metal contribution to $z_{\text {eff }}(0), z_{\text {eff, met }}(0)$, is also rising. During this entice time, the densicy profiles obtained from Thomson scattering are flat over most of the discharge. The metallic contribution to $z_{\text {eff }}$ begins to increase at approximately $560 \mathrm{~ms}$, about so to $60 \mathrm{nls}$ after the onset of the H-mode; at first it is negligible ( $z_{\text {eff, met }}$ ) $0.2)$, but it then rather quickiy reaches the final value of over 2.6 . The very last point in $z_{\text {eff, met }}$ and $z_{\text {eff }}$ necessarily contains a large uncertainty due to the low $k T_{e}$ values, and the actual values are most probably smaller than indicated. Simultaneous with the increase in $z_{\text {eff,met }}(0)$ and the increase in density, observe a steep decrease in electron temperature which drops from $1.2 \mathrm{keV}$ to $0.55 \mathrm{keV}$ and is more than expected from the clensity increase. We ascribe this drop in temperature to the accumulation of the metals in the center of the discharge and the resulting radiation collapse in the center, Very gimilar nonaccumulatjng discharges, with a similar increase 
in density, do not show this decrease in electron temperature. The indication that this is a thermal collapse due to excessive impurity radintion comes from direct bolometric measurements [11] and estimates of the time-evolving central radiation density derived froni the PKA measurements of all major metallic impurities ( $\mathrm{Ni}, \mathrm{Fe}, \mathrm{Cr}$, and $\mathrm{Ti}$ ) coupled with coconal equilibrium cooling rates [12]. Both measurements indicate central radiation losses of $1-5 \mathrm{w} / \mathrm{cm}^{3}$, which is comparable to or larger than the local electron heating power. Radiation losses due to central low-z impurities, deriged from $P H A$ soft $X-r a y$ continuun measurements, are much smaller and increase more slowly in time, confirming that the accumulation of low-z impurities is less severe [5!.

That the impurity accemulation phase in the discharge is preceded by the impurity influx from the outside is clearly seen in Pig. 2, where the soft $x-$ ray diode enhancement factor $\xi$ is plotted against an expanded time scale and for a range of the diode tangency heights $z(z=\varepsilon r, \varepsilon$ is the elongation). These data showT ware obtained from the vertically scanning scft $\mathrm{X}$-ray diode array. The enhancement factor $\xi$ is defined as a ratio of the diode signal to the signal of the same dinde if the slasma was purely hydrogen:

$$
\xi(z, t)=\frac{U_{D}(z, t)}{\int d 1 \int d E_{0} \varepsilon_{H}(E, 1) \cdot F(E) \cdot R(E)} .
$$

Here $U_{D}(z, t)$ is the measured diode signal. It represents the continuum and the line radiation (mostiy L-lines) from metallic impurities. The part of $\xi$ which cones from the continuum ragiation is equivalent to the usual. PHA enhancement factor $5[13] . \varepsilon_{H}$ is the hydrogen bremsgtrahlung emissivity, $F(E)$ is the transmission function of the filter in front of the diode, $R(E)$ is the resoonse function of the diode inciuding the diode/plasma geometry, $E$ is the photon energy, and fdl is the line of sight integral. The arrows in Fig. 2 
show the times of the onset of the increase in signal due to the increasing influx. It takes about $20 \mathrm{~ms}$ for the impurities from $z=30 \mathrm{~cm}$ to reach the center of the discharge and indicates a surge in the medium-z inpurity influx before the onset of accumulation. A gimilar increase in influx is seen in the SPRED VUV lines for lower ionization states of metallic impurities [6], but without spatial resolution.

Very soon after the increased influx has propagated from the outer diodes toward the central diodes, one observes an almost simultaneous increase of radiatinn orer the whole profile, followed oy a peaking of the profile. This is seen quite clearly in Fig. 3 , where the riode enhancement factor $\xi$ is plotted against the radial position (or height) for varions times. The central enhancement factor increases by a factor of 50 , whereas at $z=30$ s increases only by a factor of 4 , indicating a very strong central peaking of metallic impurities. The $\xi$ profiles are considerably broader than the spatially resolved $n_{T i}$ profile measurements from the PHA (shown in Fig. 4) because they are strongly weighted by a lower energy continuum radiation, which has braader profiles than the titanium $\mathrm{k}_{\alpha}$ - line radiation at the relatively high energy of $4.5 \mathrm{keV}$ or $\mathrm{n}_{\mathrm{Ti}}$ itgelf. In adaition, the profiles are broadened by strong metallic I-line radiation, which radiate at rather low energy and therefore have a radiation peak in a shell somewhat off the center of the discharge. The titanium central density increases during the influx and accumulation phase by a factor of 100 but the increase at $z=30 \mathrm{~cm}$ is much smaller ionly a factor of 10). The $n_{T i}$ profiles of Fig. 4 are obtained from the PHA measurements, but a similar peaking and enhancement of the central medium-z impurity density is seen by SPRED and the bolometer radiation profiles. The bolometer radiation profiles and theit absolute intensity in the plasma core agree quantitatively very well with the metallic impurity 
profiles (titanium, chrome, iron, and nickel) obtained from the soft $\mathrm{X}$-ray PHA and VUV measurements $[5,11]$. Note also, that the $n_{T i}$ profiles of Fig. 4 additionally support the claim that most of the $\mathrm{n}_{\mathrm{Ti}}$ increase is due to influx from the edge of the plasma. By comparing $\mathrm{n}_{\mathrm{Ti}}$ profiles at $0.51 \mathrm{~s}$ and $0.69 \mathrm{~s}$, one can easily show that most of the titanium iscrease inside the minor radius of 22.5 cm can only come via the influx from the edge $(x \geq a)$ and not via influx from the region between $22.5 \mathrm{~cm}$ and $\mathrm{a}=32 \mathrm{~cm}$ by profile rearrangement alone. Otherwise, we would have to postulate an unrealistic, very high density titanium shell between $22.5 \mathrm{~cm}$ and the edge of the plasma to account for the observed increase in the core region.

Almost an identical H-mode discharge, having the same electron density, beam power, and plasma currert, can occur without any accumulation by careful tailoring of the gas feed and other discharge parameters, In that case there is no significant medium-z influx, and the metallic impurity density and the $\xi$ factor stay at the level of $t=0.56 \mathrm{~s}$ of Fig. 4. The only difference between these two discharges is that the $z_{\text {eff }}$ at the onset of neutral beam injection is somewhat higher in the accumulation case. This dependence of the accumulation on $z_{e f f}$ and its control by gas puffing is fully described in other papers [5 - 6]. There it has been shown that the impurity caefficient is proportional to the $z_{\text {eff }}$ value at the center of the discharge and that a small increase in gas puffing can prevent the central accumulation by lowering the impurity influy and $z_{\text {eff }}(0)$.

IV. Impurity Transport Analysis Utilizing PHA Data If the spatial and temporal variation of the density of a particular impurity ion species is known in detail, it is then straightforward to find the temporal and spatial behavior of the particle flux for the case of 
toroidal and poloidal symmetry. We have already show 2 measurements of such densities utilizing soft X-ray PHA for the four most ocmmon, medium-Z PBX plasma impurities: titanium, chrome, iron, and nickel. In this section we show how to obtain the impurity flux from the PHA density measurements and, having the flux and assuming that in the most general case it contains a diffusive and a convective term, how to get the diffusion and convection coefficients.

He start with a continuity equation for a single impurity in the sourcefree plasma core

$$
a n / a t+\nabla \cdot r=0,
$$

where $n=\sum_{q} n_{q}$ is the impurity density and $\Gamma=\sum_{q} \Gamma$ is the impurity flux, $n_{q}$ is the ion density of charge state $q$, and $\Gamma_{q}$ is the flux of ions of charge state q.

If $\Gamma$ is assumed to be constant on nested circular cross-sectional toroidal surfaces of minor radius $r$, the volume integration of $E q .2$ over the nested toroidal volumt with minor radius $r$ yields the particle flux

$$
[(r, t)=-1 / r \partial / \partial t\{J n(r, t) r d r\}
$$

When the impurity density $n(r, t)$ is measured, we call obtain the impurity particle flux F by utilizing Eq. (3). Fhis flux can be either diffusive or convective, and in the most general case we assume that it contains both terms :

$$
\Gamma(r, t)=-D(r, t) \quad \nabla n(r, t)+v(r, t) n(r, t),
$$


where $\delta$ and $v$ represent an unknown diffusion coefficient and convective velocity, respectively, which for simplicity we assume to be independent of charg? state $q$. Thus, measurements of $n(r, t), \nabla n(a, t)$, and $\Gamma(r, t)$ as a function sf radils (or time) allow evaluation of the local values of $D$ and $v$ as a function of time (or radius). That is, the simultaneous equations

$$
\begin{aligned}
& r_{1}=-D n_{1}+v n_{1} \\
& r_{2}=-D \nabla n_{2}+v n_{2} .
\end{aligned}
$$

where 1 and 2 denote $n, \nabla r$ ard $\Gamma$ values at either $r_{1}$ and $r_{2}$ with $t=$ constant or $t_{1}$ and $t_{2}$ with $I=$ constant can be usea to solve for the diffusion coefticient and convection speed. The explicit solutions for $D$ and $v$ from the two linear equations given above are:

$$
\begin{aligned}
& \mathrm{D}(\mathrm{r}, \mathrm{t})=\frac{\frac{\Gamma_{1}}{n_{1}}-\frac{\Gamma_{2}}{n_{2}}}{\frac{\nabla n_{2}}{n_{2}}-\frac{\nabla n_{1}}{n_{1}}} \\
& v(r, t)=\frac{\frac{r_{1}}{n_{1}} \frac{\nabla n_{2}}{n_{2}}-\frac{\Gamma_{2}}{n_{2}} \frac{\nabla_{1}}{n_{1}}}{\frac{\nabla n_{1}}{n_{2}}-\frac{\nabla n_{1}}{n_{1}}} .
\end{aligned}
$$

Comparison of the values of $D$ and $v$ obtained by fixing either $r$ of $t$ allows an evaluation of the sensitivity of the results to the spatial and temporal resolutions and uncertainties of the measurements. The advantage of this method is obvious: averaging over two measurement points provides local measurements of both $D$ and $v$. Of rourse, substantial uncertainty can arige in 
the results due to measurement exrors in the density gradient $\nabla n$. The resuzting uncertainty in $D$ and $v$ is even higher than the relative error in 任 since the denominator in Egs. (6) and (7) are usually a fraction of tice magnitude of the individual $\nabla n / n$ terms. An addition: drawback to the use of this method for these particuiar data is that it can be applied only at electron temperatures high enough to observe $k_{a}$-line radiation. In spite of such uncertainties, this analysis approach nonetheless allows semiquantitative transport coefficients to be derived from relatively straightforward measurenents. Diffusion coefficient and convective velocity profiles obtained with this method are average values, averaged over a few charged states which are dominant at the particular radial position. One can, therefore, interpret these values as the local coefficients for the local average charge state. The application of this method is demonstrated for the case of the titanium B-mode accumulation by using the data in Fig. 4. To get the particle flux the data of Fig. 4 were smoothed both in time and radius. The radial dependence is represented by a smooth Gaussian profile: through two measured points at a fixed time to allow evaluation of $\Gamma$ and $\nabla n$. Because we have only three reasured points in $r$, we use two Gaussians and fit the first from $r=0$ to $r=11.3 \mathrm{~cm}$ and the second from $r=11.3 \mathrm{~cm}$ to $r=22.6 \mathrm{~cm}$. This fitting results with a break point at $11.3 \mathrm{~cm}$, where the density grauicit is discontinuous in the model profiles. The particle flux obtained by this fitting of the titanium density data in Fig. 4 is shown in Fig. 5. The rate of increase of the titanium flux is higher near the center of the discharge, iut starts to show sone saturation effect late in time. Further out in the discharge, this flux increase is slower and the last point even shows a decreage in inward flux, which ig an indication that the influx is slowed but that the arisal accumulation still persigts. The two curves for $11.3 \mathrm{~cm}$ are 
obtained from the two different Gaussians valid for the point at $r=11.3$ cm. The differenc; between these two curves is the indication of the error intruduced by the modelled discontinuity at $11.3 \mathrm{~cm}$.

Using the titanium flux results of Fig. 5 and Eqs. (6) and (7), the values for $D$ and $v$ can be obtained for three radial positions $(r \approx 6,19$, and $17 \mathrm{Crl}$ and as a function of time. Because $\mathrm{n}_{\mathrm{Ti}}$ is measured at only three radial positions in this first experiment, the errors in $n_{i j}(x)$ are large and the knowledge of the local values of the density gradients is eve! poorer, resulting in very large uncertainties in $D$ and $y$. Moreover, there are other, but probably wedker sources for errors in $\mathrm{n}_{\mathrm{Ti}}(r, t)$ evaluation: Abel inversion, poorly known elongation in the central part of the plasma, and possible poloidal asymetries. Nevertheless, the trends in the radial and the temporal behaviot of $D$ and $v$ can oe established. The values of $D$ near the center $(x)$ $6 \mathrm{~cm}$ ) is ratler $10 \mathrm{w}$, of the orjer of $500 \mathrm{~cm}^{2} / \mathrm{s}$, and axound $2000 \mathrm{~cm}^{2} / \mathrm{s}$ further out at $r$ : $17 \mathrm{~cm}$. The inwara convective velocity is also a function of radius; near the center its value lies between -100 and $-200 \mathrm{~cm} / \mathrm{s}$ and around $-800 \mathrm{~cm} / \mathrm{s}$ outside at $r=17 \mathrm{~cm}$. The convective velocity seems to increase faster than linearly with radius. To investigate the sensitivity of these results to model assumptions we have used several other fitting prodedures, (e.g. single Gaugsian), and have found that the resulting values of $D$ and $v$ are indeed somewhat different but again the diffusion coefficient is of the order of $1,000 \mathrm{~cm}^{2} / \mathrm{s}$, while the convective velocity scales faster than linearly with radius and has values of a huodred to several thousands $\mathrm{cm}^{2} / \mathrm{s}$, depending again on the radial position. These values are in a very good agreement with Ida's values [5] obtained earlier for the same discharges and utilizing spectroscopic vUV measurements and a time-dependent one-dimensional transport code (MIST) [14]. In most cases the value of the globol impurity 
diffusion coefficient in a tokamak discharge has been found to be substantialiy higher - about $10,000 \mathrm{~cm}^{2} / \mathrm{s}$ - and only in a few accumulating cases does one find lower values $[1,2,5,7]$. For chese accumulating H-mode discharges in PBX Ida et ai. [5] have shown that these coeficients are not inconsistent with simple neoclassical values $[5,6]$, and these results indicate that the main driving term for the inward convection is the impurityimpurity collisions.

This leads to an interesting speculation that other cases of impurity accumulation, such as discharges with counter neutral beam injection, may be due more to the increased impurity ixflux axiven by counter injection [15], which in turn induces accumulation, than to rotation effects themselves. In any case, the present studies on PBX emphasize the ned for sinultaneous detailed evaluation of all impuricy spesies in a given discharge to even begin to determine relevant dynamic processes, even in the case where nonclassical transport appears to be negligible.

Acknowledgment

This work was supported by U.S. Department of Energy Contract DE- ACO2-76-CHO-3073. 
References

[1] R.C. Islex, Nucl. Fusion 24,1599 (1984), and references therein.

[2] W. Engelhardt, O. KIueber, D. Meisel, H. Murmann, S. Sesnic, G. Fussmann, E. Glock, N. Gottardi, F. Karger, G. Lisitano, H,H. Mayer, F. Wacner in Dlasma Physics and Controlled Nuclear Fusion Research (Proc. 7 th Int. Conf., Innsbruck, 1978, IAEA, Vienna, (1979), Vol. 1, p. 123.

[3] M. Keilhacker, G. Fussmann, G, von Gierke, G. Janeschitz, M. Kornherr, K. Lackner, E..F. Muelier, P. Smeulders, F. Wagner, G. Becker, K. Beinhardi, U. Ditte, A. Eberhag^n, O. Gehre, J. Gernhardt, E. Glock, T. Grave, o, Gruber, G. Haas, M. Hesse, F.Karger, S. Kissel, O. Klueber, G. Lisitano, H.M. Mayer, K. McCormick, D. Meisel, V. Mertens, H. Murmann, H. Niedermeyer, พ. Poschenrieder, E. Rapp, F. Ryter, F. Schneider, G. siller, F. Soeldner, E. Speth, A. Staebler, K.H. Steuer, 0 . vollmer in Plasma Physics and Controlled Nuclear Fusion Research (Proc. 10th Iritern. Conf., London, 1984), IAEA, Vienna, (1985) Vol. 1, p. 71.

[4] K. Ida, R.J. Fonck, R.A. Hulse, and B. Leblane, Princeton Plasma Physies Laboratory Report PPPL-2264 (1985); Plasma Phys. Cont. Fusion, (in press).

[5] K. Ida, R.J, Fonck, S. Sesnic, R. Hulse, and B. LeBlanc, Princeton Plasma Physics Laburatory Report FPPL-2313 (1986).

[6] K. Ida, Ph.D. Thesis, University of Tokyo, Dept. of Physios (1985).

[7] F.H. Sequin and R. Petrasso, Phys. Rev. Lett. 51, 455 (1983).

[a] R.D. Petragso, K.H. Wenzel, J.E. Hopf, D.J. Sigmar, M. Greenwald, J.L. Terry, and J. Parker, MIT, Plasma Fusion Center Report PEC/JA-85-41 (1985). 
[9] G.L. Jahns, S. Ejima, R.J. Groebner, N.J. Brooks, R.K. Fisher, C.L. Hsieh, T.S. Taylor, J.C. Wesley, N. Fujisawa, T. Sugawara, Nucl. Fusion $22,1049(1982)$.

[10] M. Okabayashi, P, Beiergdorfer, K. Bol, D. Buchenauer, M.S. Chance, P. Couture, H. Eubank, H. Fishman, R.J, Fonck, G. Gammel, R. Goldston, B. Grek, R.C. Grimm, W. Heidbrink, K. Ida, S. Ishida, K. Itami, R. Izzo, K. Jaehnig, S.C. Jardin, D. Johnsan, R. Kaita, S. Kaye, H. Kugel, B. LoBlanc, J. Manickam, K. McGuire, D. Mueller, D. Monticello, W. Park, M. Reusch, G. Rewoldt, G. Schmidt, S. Sesnic, W. Stodiek, H. Takahashi, w. Tang, F. Tenney, R.B. White, in Plasma Physics and Controlled Nuclear Fusion Research (Pxac. 10th Intern. Conf., London, 1984), IAEA, Vienna (1985) Vol.I.. P.229.

[11] S. Paul, R.J. Fonck, and G. Schmidt, J. Nucl. Mater. (in press).

[12] D.E. Post and R.v. Jensen, Atomic Data and Nuclear Data Tables, 20; 397 (1977).

[13] S. von Goller, w. Stodiek, H. Fishman, S. Grebenshchikov, and E. Hinnov, Nutcl. Fusion 15, 301 (1975).

[14] R.A. Hulse, Nuclear Technol. Fusion 37, 259 (1983).

[15] S. Suckewer, A. Cavallo, S. Cohen, C. Daurnney, B. Denne, E. Hinnov, J. gosea, R. Hulge, D. Hwang, G. Schilling, B. Stratton, R. Wilson, Nucl. Fusion $24,315(1984)$. 
Figure Captiong

Fig. 1. Accumulation and thermal collapse: Time development of the electron temperature, $k T_{e}$, at three vertical positions $(z=0,15$, and $30 \mathrm{~cm})$, central electron density, $n_{e o}$ ' central $z_{\text {eff }}$ and $z_{\text {eff, met (contribution of metallic }}$ impurities to $\left.z_{\text {eff }}\right)$. Shaded area indicates staggered firing of three neutral beams.

Fig. 2. Partfcle influx: Time evolution of the Si-diode enhancement factor at different vertical tangency heights 2 . The enhancement starts to rise outside $(z=30 \mathrm{~cm}$ ) and propagates inward (arrows) in about $20 \mathrm{~ms}$. The enhancement factor $\xi$ represents the degree of 1mpurity contamination of the plasma. For pure hydrogen plasma $\xi=1$. The dashed lines separate the vertical scaleg for different diode tangency heights z. S.T. denotes a sawtooth.

Fig. 3. Accumulation: Time evolution of the si-diode enhancement profile. Peaking starts s,lightly before $0.59 \mathrm{~s}$.

Fig. 4. Thtanium accumulation: Time development of the titanium density profile as measured by the soft X-ray PHA. Double values at 0.51 and $0.54 \mathrm{~s}$ indicate the uncertainty of $\mathrm{n}_{\mathrm{T} i}$ measurement at early times, Measurements were made at $z=0,15$, and $30 \mathrm{~cm}$ (corresponding to $r=0,11.3$, and 22.6 cm with plasma enge at $a=32 \mathrm{~cm}$ ). $z$ is the vertical and $r$ the horizontal coordinate of the vean shaped plasma, PHA measurement of $\mathrm{n}_{\mathrm{ri}}$ beyond $r=22.5 \mathrm{~cm}$ is not possible becauge the electron temperature is too low to excite the titanium $\mathrm{k}_{\alpha}$ ines. 
Fig. 5. Time evolution of the titanium particla flux for three radial positions. Two gaussians are used to describe titanium density profiles up to and beyond $r=11.3 \mathrm{~cm}$ resulting in a double value at $11.3 \mathrm{~cm}$. Each curve is labelled by the radial zone used in the model. 1 indicates $r=0$ to $11.3 \mathrm{~cm}$ and 2 indicates $x=11.3$ to $22.6 \mathrm{~cm}$. 

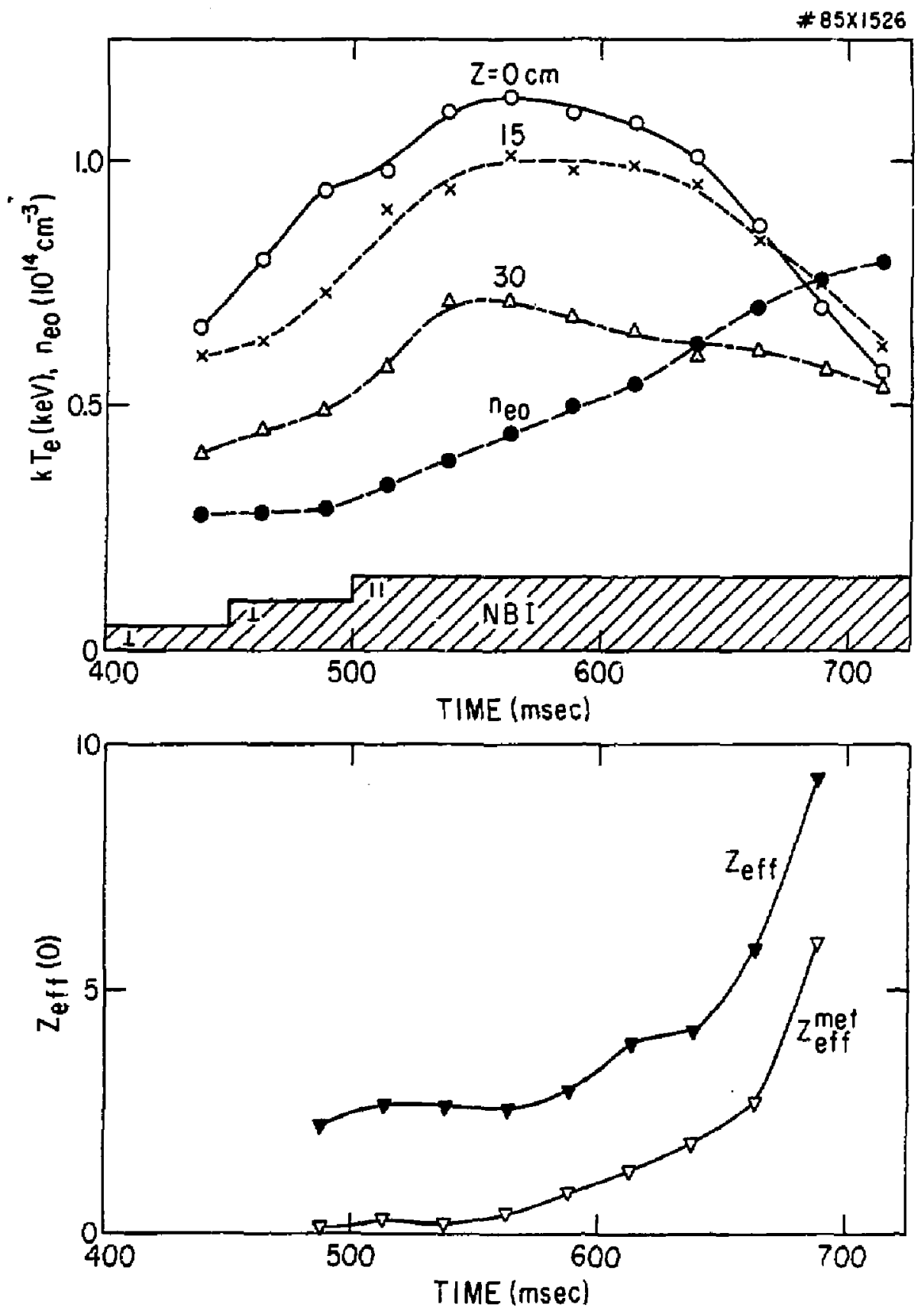

Fig. 1 


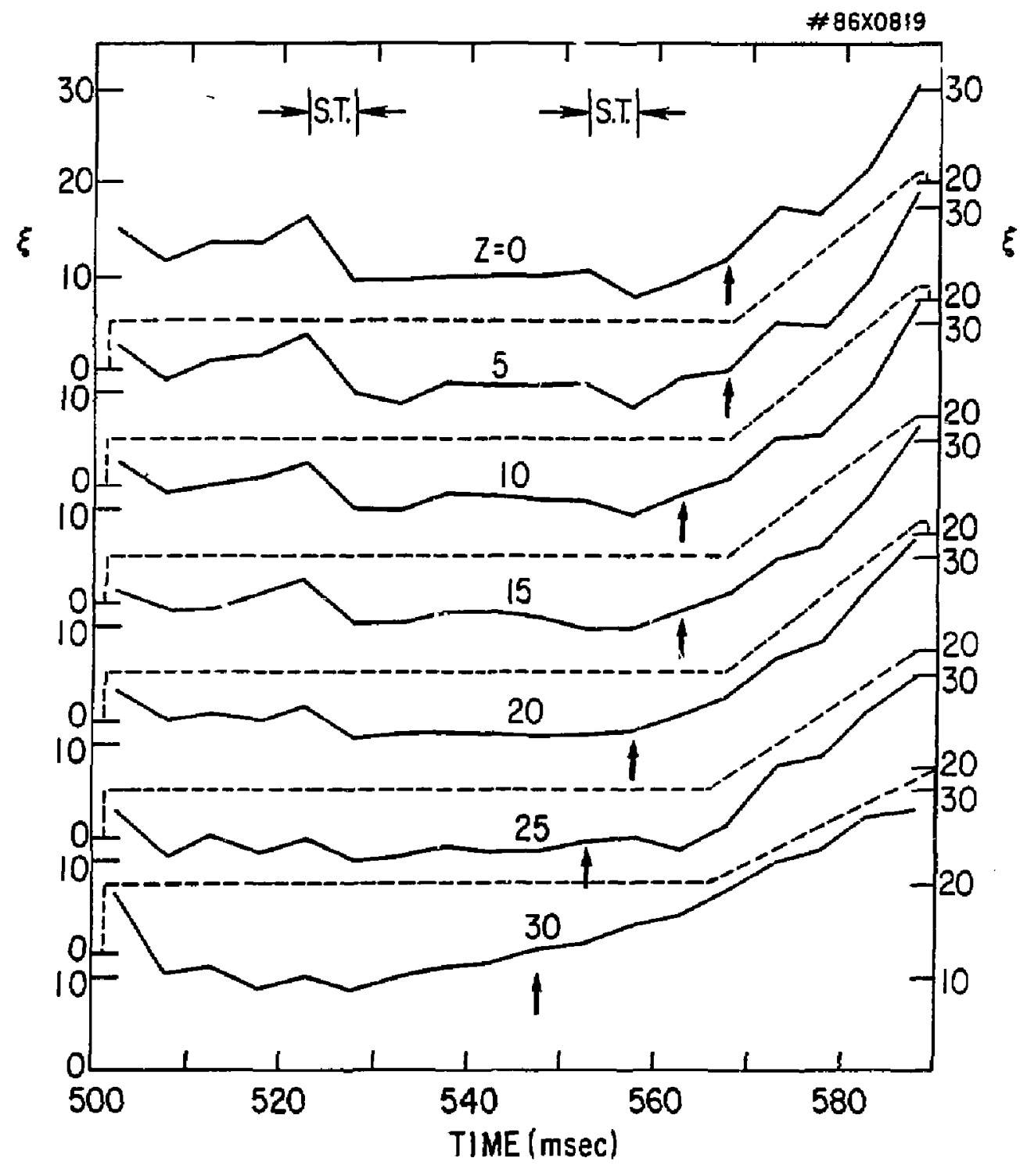

Eig. 2 


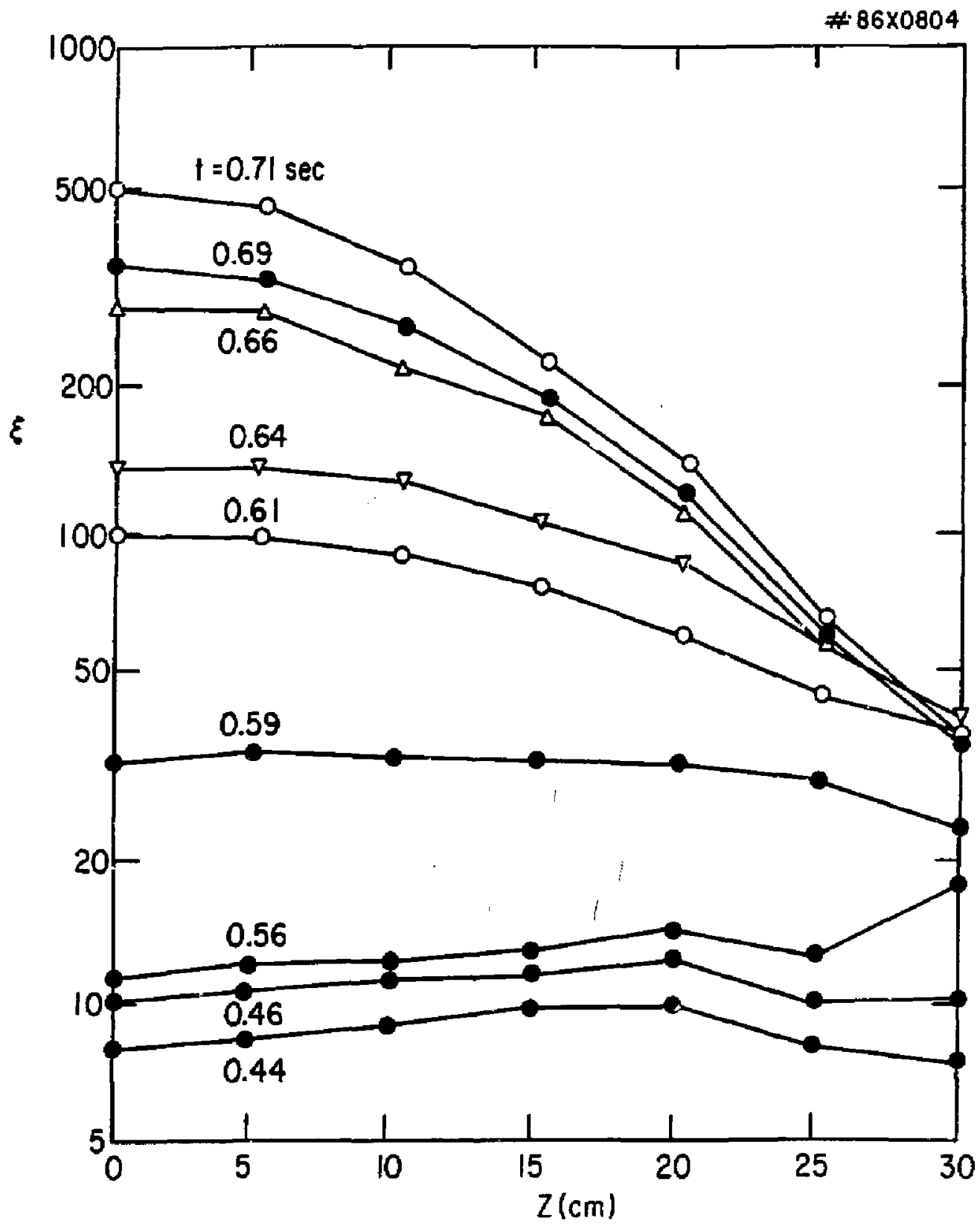

Fig. 3 

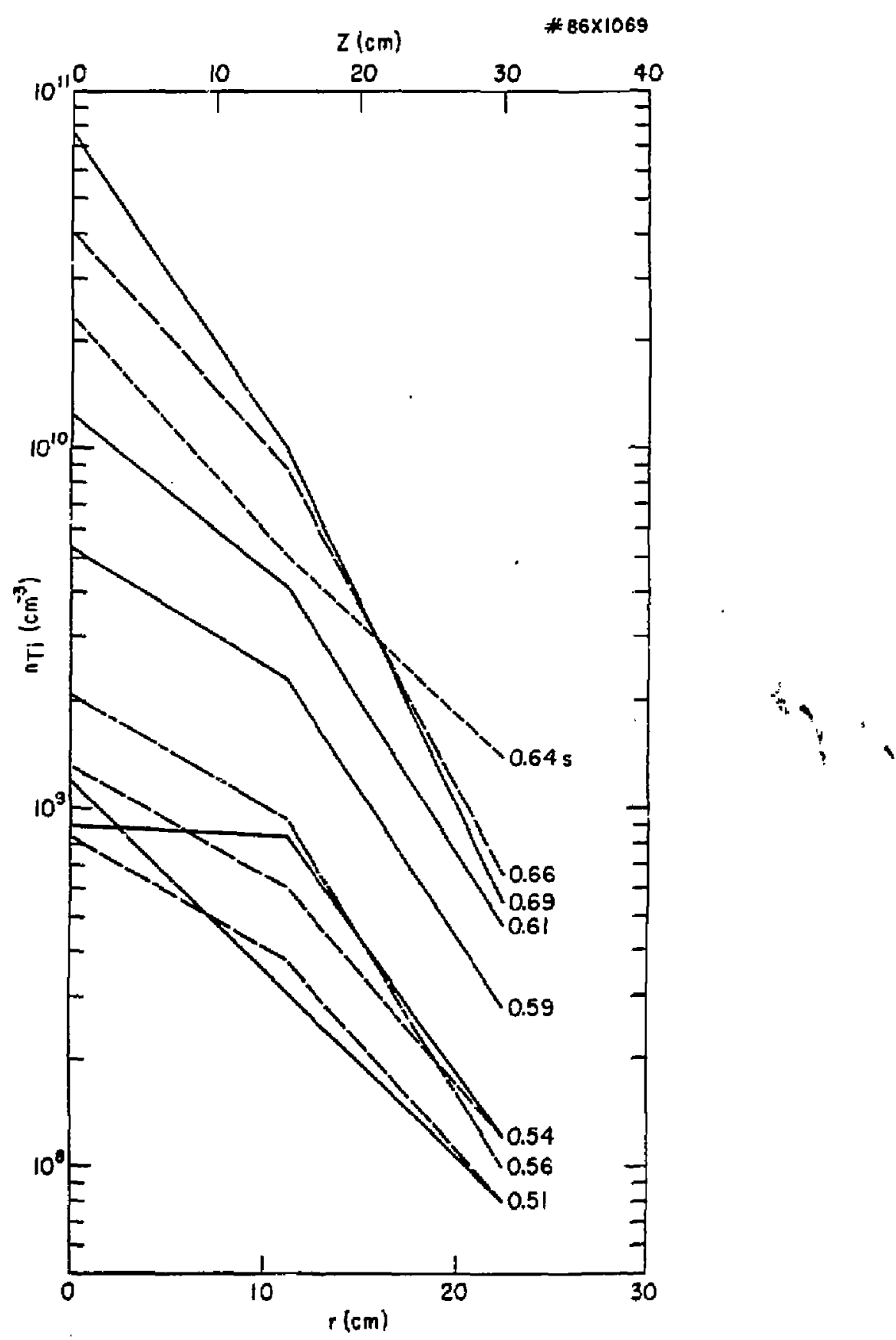

Fig, 4 


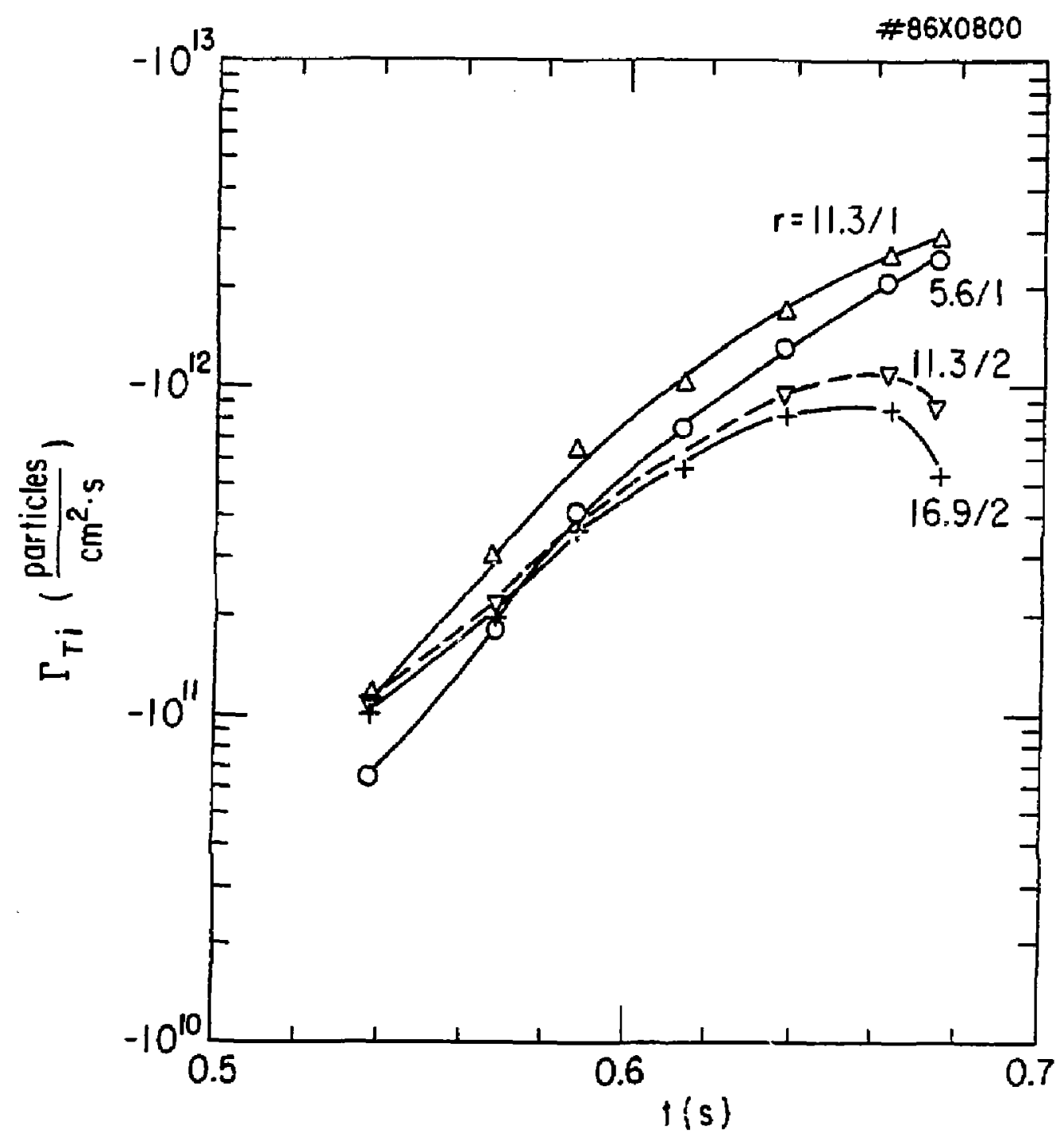

Fig. 5 
Plasma Ras Lab, Austra Nat'l Linfv, AUSTRAliA

Dr. Frank J. Postonl, Unlw of Wollongong, AUSTrial IA

Prof, I,R. Jones, Filinders UnIW.., auSTRALIA

Prof, M.H. Bronnan, UnIV Sydnoy, AUSTRALIA

PFor. F. Cap, Inst Theo Phys, AUSTRIA

M. Goossens, Astronomisch instltuut, BELGILA

Prof, R, Bouclque, Lainratorlum voor Matuurkunde, BELGIUM

Dr. D, Palumbo, Dg XII Fuston Prog, BELGIuH

Ecole Royale Milltalre, Lab de Phys Plasmas, BELGIun

Dr. P.H. Sakanaka, UnIv Estadual, BRRIL

ib. Doc. Div., Instituto do Posquisas Espaciaia, BRR IL

Dr. C.R. James, Unir of Al berta, CANADA

Prot. J. Telchmann, Unly of Montreal, CANhDA

Or. H.M. Skarsgard, Unlv of Seskatchewan, CANADA

Prot. S.R. SreenIvasan, UnIvarsity of Calgary, CAMADA

Prof. Tudor $W$. Johnston, INRS-Energle, CANNOA

Dr. Hannes Barnard, UnIv British Columbla, CANAOA

Dr. M.P. Bachynskl, log Technologles, Inc., CANADA

Chalk RIver, Nuct Lab, CAMADA

Znengwu LI, 5W Inst Physles, CHINA

Library, Tsing Hua University, CHINA

Librarlan. Institute of Physlcs, CHINA

Inst Plasme Phys, Academia Sinlca, CHINA

Or. Pator Lukac, Komenskeho Univ, ZECHOSLOVAKIA

The Librarlan, Cutham Loboratory, ENGLAND

Prot. Jchatzman, Observatolro do NIco, FRANCE

J. Rodet, CEN-BPG, FRANCE

JET Reading Room, JET Jolnt Undertaking, ENGLAND

M Dupas Library, M Dupas Llbrary, FRANCE

Dr. Tom Mual, Academy BIbl lographic, HONG KONG

Proprint Llbrary, Cent Res Inst Phys, Hukgary

Or. R.K. Chinajlanl, Vikram Unlv. IMDiA

Or. B. Dasgupta, Saha Inst, INOIA

Dr. P. Kan, Physfcal Research Lab, INDIA

Dr. Phlllip Rosenau. I srael Inst Tech, ISRAEL

Prof. 5. Cuporanan, Tol Aviv UnIvarsity, ISAaEL

Prat, G. Rostogn 1, UnIV DT Padova, ITALY

LIararian, Intil Ctr theo Phys, ITALy

MIss Clelle De Palo, Assoc EUAATOM-EhEA, ITALY

BIbl latoco, del GNA EIRATCH, ITALY

Dr. H. Yamato, Tosnl ba Res \& Dev, JAPAN

DIrwc, Dept. Lg. Tokamak Dev, JAERI, JAPAN

Prot. Nobuyukl Inoue, University of Tokyo, JAPAN

Resourch Info Conter, Nagoye University, JAPAN

Prot, Kyojl NIshikawa, Unly of Hiroshime, JAPAN

Prof. Slgeru NorI, JAERA, JAPAN

Prot. S. Tanaka, Kyoto Unlversity, JAPAN

LIbrary, Kyoto In Ivers Ity, JAPAN

Prot. I chl ro Kawakanl, NIhon UnIY, JAPAN

Prot. Satoshi Itoh, Kyushu UnIversity, JAPAN

Dr. D.t. Chol, Adv. Inst Sel a Toch, KOREA

Tech Into DIvIsIon, KAERI, KOREA
Blol lothesk, Fom-Inst Voor Plasma, NET!iERLANDS

Prot. B.S. Liloy, University of Walkato, NEW ZEALANO

Prof. J.A.C. Cabral, Inst Superfor Tesn, PORTJGAl

Or. Octavlan Petrus, ALI CLRA UnIversity, ROMANIA

Prol. M.A. Hellberg. Unlverslty of Natal, SO AFRICA

Or. Johon do Villlers, Plasma Physics, Nucar, SO AFRICA

Fusion Div. LIbrary, JEN, SPAIN

Prof. Hans Wilhelmson, Chalmers Unly Tach, SWEOEN

Or. Lennart Stenflo, Unlversity of UMEA, SWEDEN

Librory, Royal Inst Tech, SWEDEN

Centre de Recherchesen, Ecola Polytech Fed, SwITZERLAND

Dr. V.T. Tolak, Kharkov Phys Teen Ins, USSR

Dr. DiD. Ryutov, SIberlan Acad SCl, USSR

Or. G.A. EIIseer, Kurchator Institute, USSR

Dr. Y,A, GlukhIkh, Inst Electro-Physlęat, USSA

Instltute Gen. Physlcs, USSR

Prof. T.J.M. Boyd, Uniy Coll lege N wales, WALES

Dr. K. Schindler, Ruhr Universitat, W. GERMANY

ASDEX Reading Rm, IPPMax+lanck-Institut fur

PlasmaphysIK, F, R.G.

Nuclear Res Estab, Julleh Ltd, W. GERMANY

Lliorarlan, Max-Planck Institut, W. GERMANY

Glbllothek, Inst Plasmaforschung, W. GERMANY

Prof. R.K. Jonev, Inst Phys, YugosLAviA 